\title{
The Forecasting Residual Life of Corroding Pipeline based on Semi-Probabilistic Method
}

\author{
Noor, N.M. ${ }^{1}$, Yahaya, N. ${ }^{2}$, Ozman, N.A.N ${ }^{3}$, Othman, S.R. ${ }^{4}$
}

\begin{abstract}
In general, the prediction of pipeline residual life can effectively assist pipeline operators to evaluate future safe operating strategies including re-inspection and appropriate maintenance schedule. As a result it can minimize the possibility of pipeline failures until it reaches its designed lifetime. A semi-probabilistic methodology for predicting the remaining strength of submarine pipelines subjected to internal corrosion based on Recommended Practice RP-F101 by Det Norske Veritas (DNV) is described in this paper. It is used to estimate the maximum allowable operating pressure of the corroding pipelines based on series of pigging data, which represents corrosion pit location and dimension. The introduction of partial safety factors in the DNV code to minimise the effect of uncertainties due to the defect sizing has improved the reliability of pipeline assessment methodology. Nevertheless, the code is still regarded as a fully deterministic approach due to its incapability of predicting the remaining life of corroded pipeline. Thus, we have added prediction capabilities to the capacity equation by introducing a standard deviation model of future defect depth. By doing so, the variation of safety factors of the capacity equation can be fully manipulated in which prediction of future pipeline residual life becomes feasible. The paper demonstrates calculation and prediction of pipeline residual life subjects to internal corrosion. The results shows the standard deviation of corrosion parameter affected the value of partial safety factor as corrosion progressing, hence amplify the conservatism of time to failure.
\end{abstract}

Keywords: Pipeline, DNV RP-F101, corrosion, probabilistic.

\section{INTRODUCTION}

$\mathrm{O}$ ffshore (marine) and onshore pipelines are one of the safest, economical and as a consequence, the most applied means of transporting oil and gas in the world nowadays. Unfortunately, the increasing number of aging pipelines in operation has significantly increased the number of accidents ${ }^{1}$. As pipeline ages, it can be affected by a range of corrosion mechanisms, which may lead to a reduction in its structural integrity and eventual failure ${ }^{1-3}$. Corrosion is an imperative form of pipeline deterioration due to aggressive environments ${ }^{4}$. Without practical and effectual corrosion prevention strategy, corrosion will continue to progress and the cost of repairing a deteriorating pipeline will escalate. Significant savings are possible by optimizing the inspection and corrosion prevention strategies ${ }^{5}$.

\section{RESEARCH PROBLEM AND METHODOLOGY}

Since corrosion is a complex process involving numerous unknown factors, the prediction task has always been a challenge to pipeline owners especially when vital information is lacking of. The inherent uncertainties embedded within metal loss data plays significant roles in reducing the accuracy of pipeline future assessment. These uncertainties are related to tool imperfect measurement, randomness of environment and variation of operational data. To cater for the uncertainties, DNV RP-F101 has incorporated safety factors which specially tailored for defect depth. Yet, the code is still regarded as a deterministic approach akin to other capacity equation such as ASME B31G and PCORRC since the safety factors represent averaged value and no variation of parameters are included in the calculation of pipeline remaining pressure. Unlike conventional safety factors, the value is dependent upon inspection tool accuracy which is defined by the dispersion of corrosion growth rate value and metal loss data. Nevertheless, the code is designed to assess the condition of the line at the time of inspection owing to constant partial safety factor. In order to predict the remaining pressure in the future, the amount of unknown uncertainties which theoretically escalate with time must be taken into account. This is reflected by the increment of partial safety factors as a function of time to represent the influence by these unknown uncertainties related to randomness of corrosion progress, environment as well as material properties ${ }^{6}$. The originality of this paper relies on the introduction of statistical-based equation which is able to estimate the new standard deviation value of possible future defect depth. In our works, we manipulate the variation of safety factors in RP-F101 to make the deterministic capacity equation capable of predicting the future growth of defects. The future metal loss data based on prediction is supposed to pose higher variation of its value compared to actual metal loss data. Hence, higher safety factors of defect depth will increase the conservatism of assessment due to rapid reduction of structure capacity which is more realistic. 


\section{Pipeline Inspection Data}

In our case study, an extensive amount of pigging data has been gathered through repeated in-line inspection activities using MFL intelligent pig on the same pipelines at different point of times. The transmission pipelines located in North Sea area used to convey crude oil and gas (multiphase line) from central offshore platform to onshore terminal. The data provides valuable information on the internal corrosion defect geometry, such as defect location, depth and length, orientation and types of corrosion regions as displayed in Table 1.

Table 1: A typical presentation of pigging data

\begin{tabular}{|c|c|c|c|c|c|c|c|c|}
\hline $\begin{array}{c}\text { Spool Length } \\
(\mathbf{m})\end{array}$ & $\begin{array}{c}\text { Relative } \\
\text { distance } \\
(\mathbf{m})\end{array}$ & $\begin{array}{c}\text { Absolute } \\
\text { distance } \\
(\mathbf{m})\end{array}$ & $\begin{array}{c}\boldsymbol{d} \% \\
\boldsymbol{w} \boldsymbol{t}\end{array}$ & $\begin{array}{c}\boldsymbol{l} \\
(\mathbf{m m})\end{array}$ & $\begin{array}{c}\boldsymbol{W} \\
(\mathbf{m m})\end{array}$ & O'clock & $\begin{array}{c}\boldsymbol{t} \\
(\mathbf{m m})\end{array}$ & Loc. \\
\hline 11.6 & 6.6 & 1016.5 & 18 & 32 & 42 & 6.00 & 14.2 & Internal \\
\hline 11.5 & 11.5 & 1033.0 & 19 & 46 & 64 & 5.30 & 14.2 & Internal \\
\hline 11.8 & 10.6 & 1043.6 & 12 & 18 & 55 & 5.30 & 14.2 & Internal \\
\hline 11.7 & 1 & 1045.8 & 13 & 28 & 83 & 5.30 & 14.2 & Internal \\
\hline
\end{tabular}

where:

Absolute distance : Distance of corrosion from start of pipeline

d\%wt : Maximum depth of corrosion in terms of percentage

$l \quad$ : Longitudinal extent of corrosion

Loc : Location of corrosion either internal or external.

O'Clock : Orientation of corrosion as a clock position.

Relative distance : Relative distance of corrosion from upstream girth

Spool length $\quad:$ Length of pipe between weld (10m to $12 \mathrm{~m}$ approximately)

$t_{t} \quad:$ Nominal thickness of pipe in pipe spool

$W \quad$ : Extent of corrosion around pipe circumference weld

\section{Assessment Practice}

In general, the degree of conservatism in regard to structural assessment is due to the implementation of safety factor in deterministic method. This safety factor is associated with load factor or resistance factor (strength of material), which is commonly found in all codes of assessment for corroded pipelines. The uncertainties subjected to structural properties, loading condition, environmental behavior and construction performance are always neglected in the calculation due to the employment of safety factor. In pipeline assessment, deterministic assessment is a straight-forward approach based on codes or developed capacity equation. Generally, the deterministic methods use lower bound data; for instance peak depth of corrosion, maximum corrosion rate and minimum wall thickness without considering the existing uncertainties 7 . Consequently, it can be over conservative in terms of safety when being implemented to pipelines containing extensive corrosion defects. For example, the prediction of future growth of corrosion defects located in the pipelines will use an average for single rate value without considering the possibility that not all defects will grow at the same rate. The averaged rate is used for the sake of simplicity owing to lack of information pertaining to environmental and material properties. Assessment method is required to determine the severity of such defects when they are detected in pipelines ${ }^{8}$. The assessment of the condition of existing oil and gas pipeline is necessary in order to protect the public, financial investment and environment from such failures. Systematic and optimized regular inspections of pipelines with state-of-the art tools and procedures can reduce significantly the risk of any undue accident caused by a lack of unawareness of the integrity of the line

\section{DNV-RPF101 CODE}

The DNV Recommended Practice for the assessment of corroded pipelines, DNV-RP-F101 was issued in 1999. RP-F101 describes two alternative approaches with different safety philosophy. The equations in RP-F101 were derived by a probabilistic calibration ${ }^{10}$, taking into account the uncertainties in defect measurements and burst capacity. The equations account directly for the accuracy in sizing the corrosion defect. The RP -F101 recommends the assessment of corroded pipelines subject to internal pressure and internal pressure combined with longitudinal compressive stresses ${ }^{10}$. Moreover, this new criterion provides an assessment procedure for single defect, interacting defects and complex shaped defects. The maximum allowed operation pressure in pipelines for a single defect is given as: 


$$
P_{p}=\frac{\gamma_{m} \times 2 \times t \times \operatorname{SMTS} \times\left(1-\gamma_{d}(d / t)^{*}\right)}{(D-t)\left(1-\gamma_{d}(d / t)^{*} Q^{-1}\right.} \leq P_{\text {mao }}
$$

where;

$$
\begin{gathered}
Q=\sqrt{1+0.31\left(\frac{L}{\sqrt{D t}}\right)^{2}} \\
(d / t)^{*}=(d / t)_{\text {meas }}+\varepsilon_{d} S t D[d / t]
\end{gathered}
$$

and;

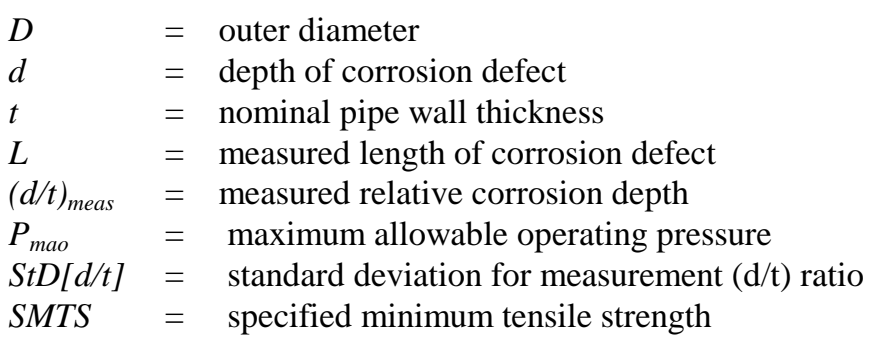

\section{Partial Safety Factor}

The partial safety factors $\gamma_{\mathrm{m}}$ and $\gamma_{\mathrm{d}}$, and the fractile value $\varepsilon_{\mathrm{d}}$ are determined from tables which depend on the safety class classification, the pipe quality, inspection method and sizing accuracy of the inspection tool ${ }^{11}$. It was given as functions of the sizing accuracy of the measured defect depth for inspections based on relative depth measurements and for inspections based on absolute depth. The safety class is specified based on Table 2 to Table 4 .

Table 2: Partial safety factor $\gamma_{\mathrm{m}},{ }^{1}$

\begin{tabular}{|l|l|l|c|}
\hline \multicolumn{1}{|c|}{ Inspection Method } & \multicolumn{3}{c|}{ Safety class } \\
\cline { 2 - 4 } & \multicolumn{1}{|c|}{ Low } & Normal & High \\
\hline Relative (e.g. MFL) & $\gamma_{\mathrm{m}}=0.79$ & $\gamma_{\mathrm{m}}=0.74$ & $\gamma_{\mathrm{m}}=0.70$ \\
\hline Absolute (e.g. UT) & $\gamma_{\mathrm{m}}=0.82$ & $\gamma_{\mathrm{m}}=0.77$ & $\gamma_{\mathrm{m}}=0.72$ \\
\hline
\end{tabular}

Table 3: Standard deviation, StD [d/t], for MFL inspection tool ${ }^{11}$

\begin{tabular}{|c|c|c|c|c|}
\hline \multirow{2}{*}{$\begin{array}{l}\text { Inspection sizing accuracy } \\
\operatorname{StD}[d / t]\end{array}$} & \multirow[b]{2}{*}{$\varepsilon_{d}$} & \multicolumn{3}{|c|}{ Safety class } \\
\hline & & Low & Normal & High \\
\hline (exact) 0.00 & 0.0 & $\gamma_{d}=1.00$ & $\gamma_{d}=1.00$ & $\gamma_{d}=1.00$ \\
\hline 0.04 & 0.0 & $\gamma_{d}=1.16$ & $\gamma_{d}=1.16$ & $\gamma_{d}=1.16$ \\
\hline 0.08 & 1.0 & $\gamma_{\mathrm{d}}=1.20$ & $\gamma_{\mathrm{d}}=1.28$ & $\gamma_{d}=1.32$ \\
\hline
\end{tabular}

\begin{tabular}{|l|l|l|}
\hline Relative sizing accuracy & Confidence level \\
\cline { 2 - 3 } & $\mathbf{8 0 \%}$ & $\mathbf{9 0 \%}$ \\
\hline Exact $\pm(0.0$ of $\mathrm{t})$ & $\mathrm{StD}[\mathrm{d} / \mathrm{t}]=0.00$ & $\mathrm{StD}[\mathrm{d} / \mathrm{t}]=0.00$ \\
\hline \pm 0.05 of $\mathrm{t}$ & $\mathrm{StD}[\mathrm{d} / \mathrm{t}]=0.04$ & $\mathrm{StD}[\mathrm{d} / \mathrm{t}]=0.03$ \\
\hline \pm 0.10 of $\mathrm{t}$ & $\mathrm{StD}[\mathrm{d} / \mathrm{t}]=0.08$ & $\mathrm{StD}[\mathrm{d} / \mathrm{t}]=0.06$ \\
\hline
\end{tabular}

Table 4: Partial safety factor, $\gamma_{\mathrm{d}}$ and fractile value factor, $\varepsilon_{\mathrm{d}}{ }^{11}$

\section{SEMI-PROBABILISTIC EQUATION}

The next section of this paper demonstrates the efforts of deriving a semi-probabilistic equation intended to calculate the future variation of corrosion defects. The term semi-probabilistic means that the proposed equation still requires averaged values (fixed value) of parameters instead of random values. Nonetheless, the estimated value is related to the probable variation of corrosion defects in the future. Moreover, the equation is derived based on the principal of variance of 
probability distribution. Hence, the terms semi-probabilistic was chosen to signify the probabilistic element within deterministic equation. The augmentation of prediction capabilities by increasing the standard deviation of predicted can be explained mathematically by referring to linear growth rate model. The corrosion rate equation can be written as:

$$
C R=\frac{d_{t_{1+1}}-d_{t_{i}}}{T}
$$

where, $T=t_{i+1}-t_{i}$ and is a constant value.

If corrosion depth $d$ is assumed statistically varied whereas $T$ is fixed, the variation of corrosion rate can be expressed as:

and, simplified into:

$$
\sigma_{C R}^{2}=\frac{1}{T^{2}} \cdot \text { variance }\left(d_{t_{1+1}}-d_{t_{i}}\right)
$$

$$
\sigma_{C R}^{2}=\frac{1}{T^{2}}\left(\sigma[d]_{t_{i+1}}^{2}-[d] \sigma_{t_{i}}^{2}\right)
$$

Therefore, the relationship between inspection time interval and the variation in corrosion growth rate can be presented as:

$$
\sigma_{C R}=\frac{1}{T} \sqrt{\left(\sigma[d]_{t_{i+1}}^{2}-\sigma[d]_{t_{i}}^{2}\right)}
$$

Since $\sigma=S t d$, therefore

$$
S t d_{C R}=\frac{1}{T} \sqrt{\left(S t d[d]_{t_{i+1}}^{2}-S t d[d]_{t_{i}}^{2}\right)}
$$

Inspection data of metal loss from MFL pig tools usually is represented as a ratio of defect depth to wall thickness, $d / t$. By replacing the exact metal loss value, $d$ with metal loss ratio, $d / t$, Equation 8 can be rewritten as follows;

$$
S t d\left[\frac{c r}{t}\right]=\frac{1}{T} \sqrt{\left(S t d\left[\frac{d}{t}\right]_{t_{i+1}}^{2}-S t d\left[\frac{d}{t}\right]_{t_{i}}^{2}\right)}
$$

By assuming the wall thickness, $t$ as a fixed value with no variation, and $c r=d / t$, the conclusive equation can be presented as

$$
S t d[d / t]_{T}=\sqrt{S t d\left([d / t]_{o}\right)^{2}+\frac{T^{2}}{t^{2}} S t d[c r]^{2}}
$$

where:

$\operatorname{Std}[\mathrm{d} / \mathrm{t}]_{\mathrm{o}}=$ Standard deviation of inspection tool in first year assessment.

$\operatorname{Std}[\mathrm{d} / \mathrm{t}]_{\mathrm{T}}=$ Standard deviation of inspection tool in the future.

$\operatorname{Std}[\mathrm{cr}]=$ Standard deviation of corrosion rate.

$\mathrm{T}=$ prediction interval in year

The equation depicts relationship between deviation of predicted data and the interval of prediction. The longer the prediction interval, the higher the variation of future metal loss, hence the higher the partial safety factors for metal loss. Table 5 shows the equations required to estimate the partial safety factors for metal loss and fractile value according to the range of metal loss standard deviation, $S t d[d / t]$. 
Table 5: Polynomial equation for partial safety factor (defect depth) and fractile value [DNV, 2004]

\begin{tabular}{|l|l|l|}
\hline \multicolumn{1}{|c|}{ Safety Factors } & \multicolumn{1}{|c|}{$\gamma_{\mathbf{d} \text { and }} \boldsymbol{\varepsilon}_{\mathbf{d}}$} & \multicolumn{1}{|c|}{ Range } \\
\hline Low & $\gamma_{d}=1.0+4.0 \mathrm{StD}[d / t]$ & $\mathrm{StD}[d / t]<0.04$ \\
& $\gamma_{d}=1+5.5 \mathrm{StD}[d / t]-37.5 \mathrm{StD}[d / t]^{2}$ & $0.04 \leq \mathrm{StD}[d / t]<0.08$ \\
& $\gamma_{d}=1.2$ & $0.08 \leq \mathrm{StD}[d / t] \leq 0.16$ \\
\hline Normal & $\gamma_{d}=1+4.6 \mathrm{StD}[d / t]-13.9 \mathrm{StD}[d / t]^{2}$ & $\mathrm{StD}[d / t] \leq 0.16$ \\
\hline High & $\gamma_{d}=1+4.3 \mathrm{StD}[d / t]-4.1 \mathrm{StD}[d / t]^{2}$ & $\mathrm{StD}[d / t] \leq 0.16$ \\
\hline
\end{tabular}

\section{RESULTS \& DisCUSSION}

Pipeline time to failure was determined using the DNV RP-F101 capacity equation (Part A), also known as a semiprobabilistic assessment ${ }^{11}$. Figures 1 to 3 show the prediction results of pipeline assessment subjected to internal corrosion from year $t_{0}$ to $t_{7}$. These predictions were based on gathered corrosion data from pigging inspection done in year $t_{0}$. From the assessment result in year $t_{0}$, the measured corrosion defect is within the acceptance criteria for all operating pressures where there are no defects exceeding the acceptance line as shown in Figure 1. Further prediction was carried out from year $t_{2}$ until $t_{7}$. The pipeline is considered fail or in critical condition due to bursting in year $t_{7}$ when the acceptance criteria for all operating pressure were exceeded by the projected defects (refer to Figure 3). Based on the results, it can be concluded that the pipeline should be inspected no later than year $t_{5}$ for every condition of operating pressure (refer to Figure 2). The acceptance line predicted by the corrosion defect in year $t_{7}$ was found to be lower than the one estimated for the $t_{0}$ prediction. This is due to the increment of uncertainties related to the averaged corrosion growth rate. Equation 10 and Table 5 were used to recalculate the abovementioned values as corrosion progress in time. Hence, reduce the maximum allowable defect length and depth.

\section{CONCLUSION}

The deterministic approach has the distinct advantage of simplicity that is capable to be applied in an entire pipeline or collection of pipelines easily. The disadvantage of the deterministic approach may often, but not entirely be linked to inaccuracies in the input data, but notably the inability to deal with uncertainties in the input data. The uncertainties of the deterministic approach are caused mainly by the averaged value of each parameter in the calculation i.e. lower bound of data. In the deterministic assessment the population of the corrosion dimension was assumed to grow at the same rate. A semiprobabilistic theory was introduced in the DNV RP-F101 code by estimating the standard deviation of inspection tool error and defect sizing. Nevertheless, the uncertainties in theory still inherently exist owing to a single or averaged value of parameters used in the calculation. These averaged or single values are unable to eliminate the uncertainties that might have occurred and increased over the years of service. Therefore, the prediction of pipeline integrity by using a deterministic assessment cannot fulfill the cost saving requirement by the operators. This leads to the condition whereby the operators have to inspect their pipelines frequently in order to obtain accurate information on pipeline condition. However, deterministic assessment is still widely used, but only to assess the current condition of the pipelines, owing to the lack of its prediction capabilities. The introduction of partial safety factors to minimise the effect of uncertainties due to the defect sizing and standard deviation model of future metal loss has improved the capability of predicting the future growth of corrosion defects deterministically. To improve prediction capability within deterministic framework, the inclusion of semi-probabilistic equation of future defect depth variation can be perceived as the right tool in reducing the stagnant state of structure resistance. In fact, structure assessment must take into account that when pipeline is aging, so does the structure resistance or capacity.

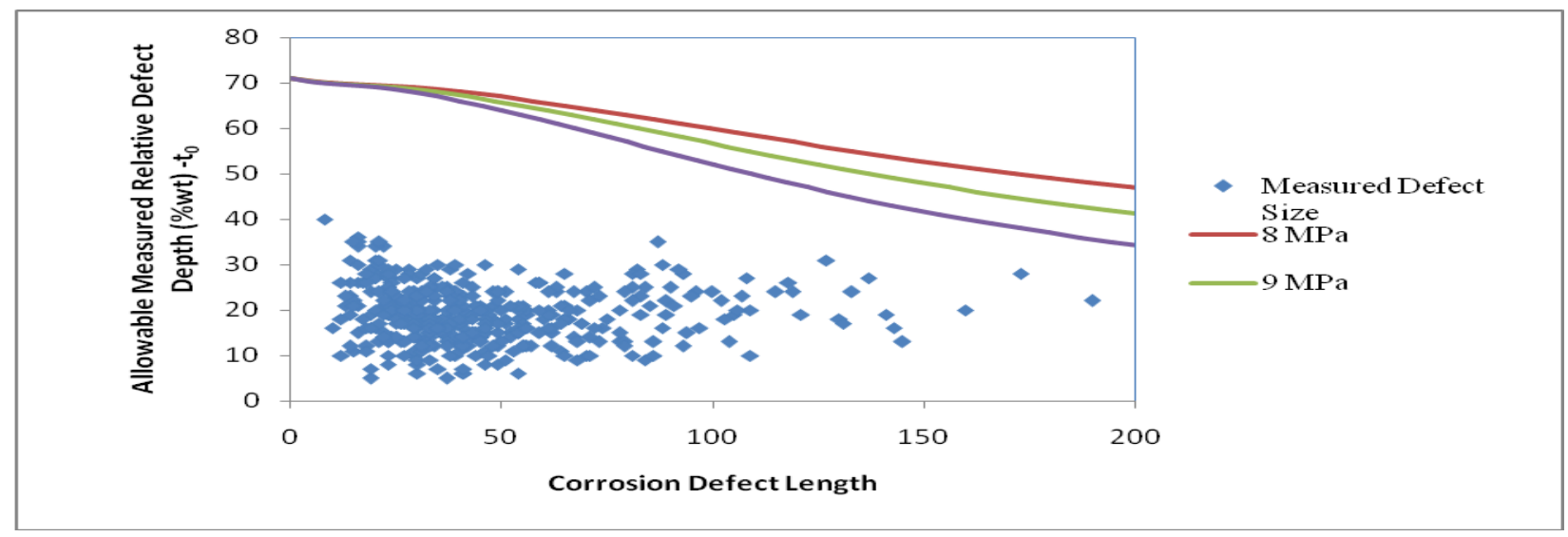

Figure 1: Projection of corrosion depth in year $t_{0}$ using DNV RP-F101 Assessment code 


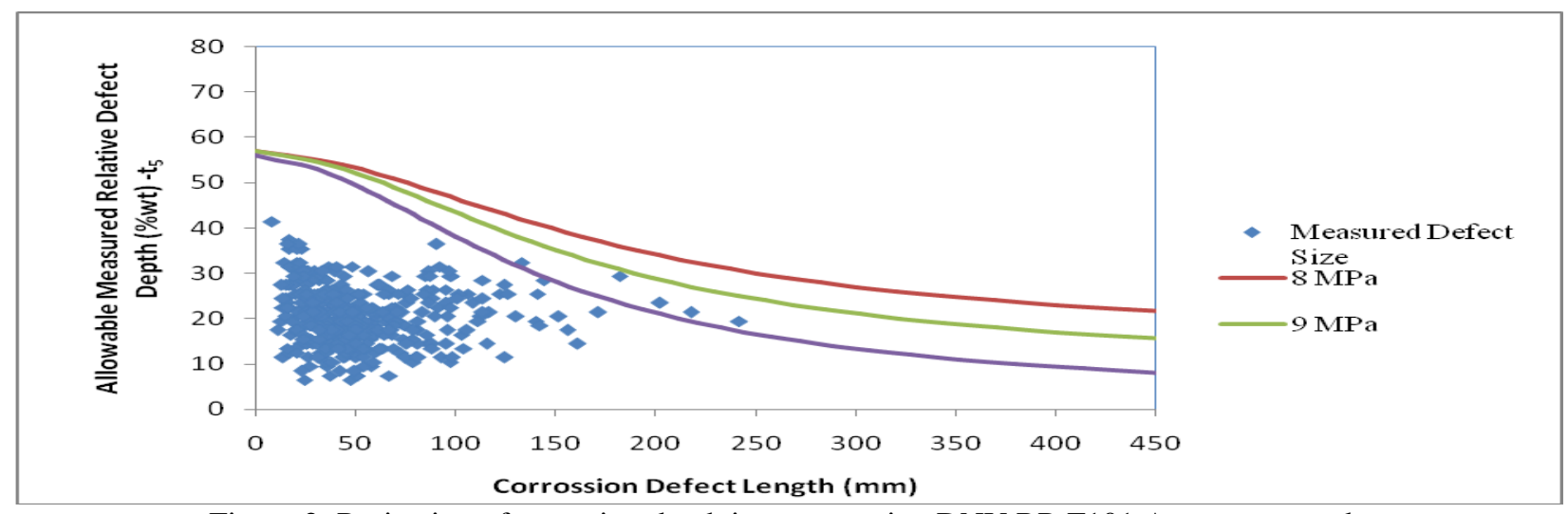

Figure 2: Projection of corrosion depth in year $t_{5}$ using DNV RP-F101 Assessment code

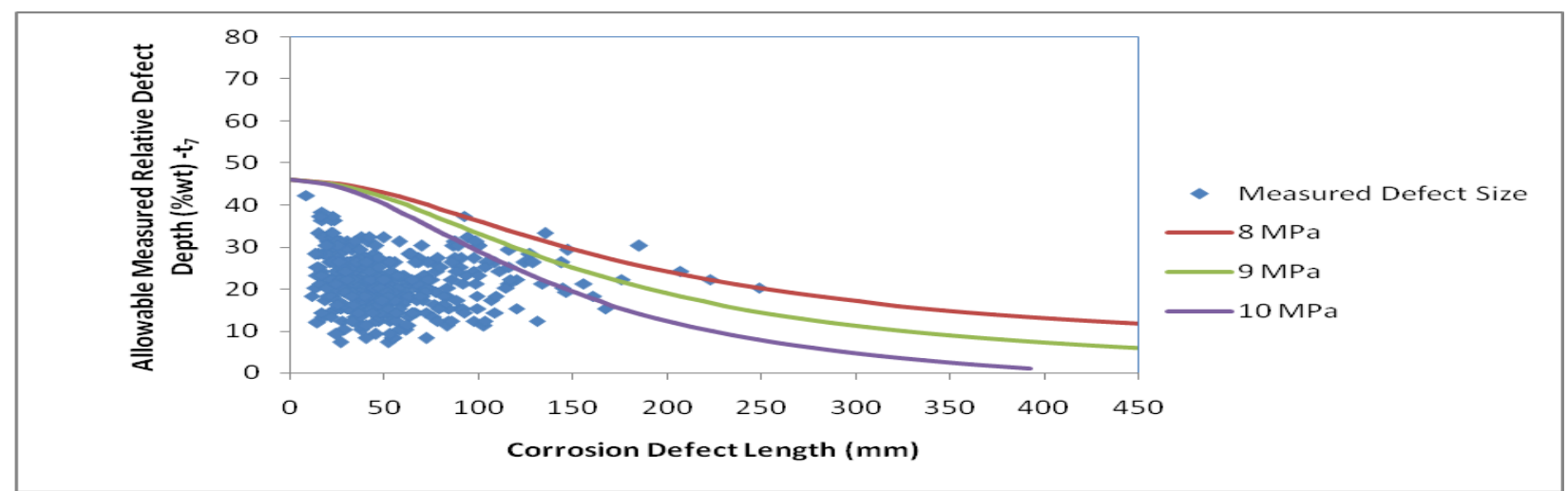

Figure 3: Projection of corrosion depth in year $t_{7}$ using DNV RP-F101 Assessment code

\section{ACKNOWLEDGEMENT}

The first author is pleased to acknowledge the Ministry of Science, Technology and Innovation, Malaysia (MOSTI) and the Ministry of Higher Education (MOHE) for the support by providing the research funds and scholarship (NSF).

\section{REFERENCES}

[1] Teixera, A.P., Guedes Soares, C., Netto, T.A., Estefen S.F. (2008). "Reliability of pipeline with corrosion defects." International Journal of Pressure Vessels and Piping. 85. 228 - 237.

[2] Ahammed, M. (1998). "Probabilistic Estimation of Remaining Life of a Pipeline in the Presence of Active Corrosion Defects." International Journal of Pressure Vessels and Piping. 75. 321-329.

[3] Netto, T.A., Ferraz U.S., Estefen S.F., (2005). "The effect of corrosion defects on the burst pressure of pipelines." Journal of Constructional Steel Research. 61, 1185-1204.

[4] Ahammed, M. and Melchers, R.E. (1996). "Reliability Estimation of Pressurised Pipelines Subject to Localised Corrosion Defects." International Journal of Pressure Vessels and Piping. 69. 267-272.

[5] Ainouche, A. (2006). "Future Integrity Management Strategy of a Gas Pipeline Uisng Bayesian Risk Analysis." $23^{\text {rd }}$ World GAS Conference.5 - 9 June. Amsterdam, Netherlands:3.3EF.05.

[6] Yahaya, N. (2000). "Risk - Based Method in Pipeline Maintenance Optimisation." Hanover, Germany: Proceeding of the Global Dialogue World Exposition (EXPO2000). Science and Technology, Thinking the future. July 11-13

[7] Yahaya, N., Noor, N.M., Din, M.M., Nor, S.H.M., (2009). "Prediction of $\mathrm{Co}_{2}$ Corrosion Growth in Submarine Pipelines" Malaysian Journal of Civil Engineering 21(1) : 69 - 81

[8] Cosham A. and Hopkins P., "The assessment of corrosion in pipelines - Guidance in the pipeline defect assessment manual (PDAM)," International Colloquium Reliability of High Pressure Steel Pipelines, pp 1-30, 2003

[9] Cosham, A., Hopkins, P., Macdonald, K.A. (2007). Best practice for the assessment of defects in pipelines - Corrosion. Engineering Failure Analysis. 14. 1245 - 1265.

[10] Bjornoy, O.H. and Marley, M.J. (2001). “Assessment of Corroded Pipelines , Past, Present and Future.” Stavanger, Norway: ISOPE 2001, Proceedings of the $11^{\text {th }}$ International Offshore and Polar Engineering Conference. June 17-22. Vol II. 93-101.

[11] DNV (Det Norske Veritas) (2004). “Recommended Practice RP-F101 for Corroded Pipelines 2004.” Norway: Det Norske Veritas. 